\title{
Relações peso-comprimento em camarões peneídeos de diferentes estoques no Sudeste do Brasil
}

Este estudo ajusta e compara as relações peso-comprimento de três espécies de camarões peneídeos (Xiphopenaeuss kroyeri, Artemesia longinaris e Litopenaeus

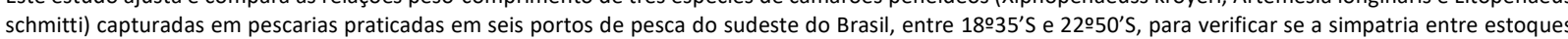
de diferentes espécies influencia o crescimento relativo. A amostragem foi realizada durante o desembarque pesqueiro em junho e julho de 2017 , a partir do volume total capturado na pescaria, representando uma parcela do estoque explorado. O comprimento da carapaça e peso foram aferidos em cada indivíduo coletado. As regressões peso-comprimento $\left(P=a^{*} C C b\right)$ foram ajustadas para machos e fêmeas separadamente, e entre os gêneros combinados. Os coeficientes alométricos (b) indicam forte alometria negativa quanto ao crescimento relativo dos camarões para todas as espécies, gêneros e estoques estudados. O peso total dos camarões aumenta a taxa relativamente menor em comparação ao comprimento do cefalotórax. Os coeficientes alométricos não diferiram entre os estoques da mesma espécie ao longo dos portos de pesca, e nem entre fêmeas e machos considerando o mesmo estoque, independentemente da presença de outra espécie no campo de pesca. Para X. kroyeri, que se distribui nos seis portos de pesca, o crescimento relativo de fêmeas, machos e gêneros combinados foi semelhante tanto onde a pesca é monoespecífica quanto na presença de outras espécies de camarões. Esse estudo apresenta o primeiro ajuste da relação peso-comprimento em estoques naturais de L. schmitti da costa dos estados do Espírito Santo (Anchieta) e Rio de Janeiro (Macaé). Recomenda-se o acompanhamento regular dos estoques para avaliar o desenvolvimento dos camarões no longo prazo e monitorar sua dinâmica de crescimento.

Palavras-chave: Relações morfométricas; Crescimento relativo; Alometria; Oceano Atlântico.

\section{Weight-length relationships in penaeid shrimps from different stocks in Southeastern Brazil}

This study adjusts and compares the weight-length relationships of three peneid shrimp species (Xiphopenaeus kroyeri, Artemesia longinaris and Litopenaeus

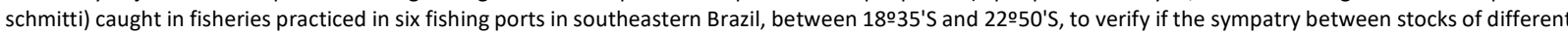
species influences its relative growth. The sampling was carried out in landings from June and July 2017, from the total volume captured in the fishery, representing a portion of the stock. The weight-length regressions $(P=a * C C b)$ were adjusted for males and females separately, and between the combined genders. The allometric coefficients (b) indicate a strong negative allometry regarding the relative growth of shrimp for all species, genders and stocks. The total weight of the shrimps increases at a relatively lower rate compared to the cephalothorax length. The allometric coefficients did not differ between stocks of the same species across the fishing ports, nor between females and males from the same stock, regardless of the presence of another species in the fishing area. For X. kroyeri, which is distributed in the six fishing ports, the relative growth of females, males and combined genders was similar both where fishing is monospecific and in the presence of other species. This study presents the first adjustment of the weight-length relationship for natural stocks of L. schmitti off Espírito Santo (Anchieta) and Rio de Janeiro (Macaé) states. Regular monitoring of stocks is recommended to assess the development of shrimps in the long term and to monitoring their growth dynamics.

Keywords: Morphometric relationships; Relative growth; Alometry; Atlantic Ocean

Topic: Conservação da Biodiversidade

Reviewed anonymously in the process of blind peer.
Received: 03/02/2021

Approved: 25/02/2021
Keltony de Aquino Ferreira (iD)

Universidade Estadual do Norte Fluminense Darcy Ribeiro, Brasil http://lattes.cnpq.br/1948328776516371 http://orcid.org/0000-0002-2005-364X

keltony.aquino@yahoo.com.br

Ana Paula Madeira Di Beneditto (iD

Universidade Estadual do Norte Fluminense Darcy Ribeiro, Brasil http://lattes.cnpq.br/8402221071942314

http://orcid.org/0000-0002-4248-9380

anapaula@uenf.br
Referencing this:

FERREIRA, K. A.; BENEDITTO, A. P. M.. Relações peso-comprimento em camarões peneídeos de diferentes estoques no Sudeste do Brasil. Revista Ibero Americana de Ciências Ambientais, v.12, n.2, p.167176, 2021. DOI: http://doi.org/10.6008/CBPC2179$\underline{6858.2021 .002 .0017}$

DOI: 10.6008/CBPC2179-6858.2021.002.0017 


\section{INTRODUÇÃO}

Os camarões peneídeos são os alvos mais importantes das capturas comerciais de crustáceos em águas tropicais (GILLET, 2008). Na costa brasileira, o camarão sete barbas, Xiphopenaeus kroyeri, é uma das espécies mais exploradas pela pesca extrativa marinha, com importância econômica para as comunidades litorâneas ${ }^{1}$, sendo o terceiro recurso mais explorado pela pesca extrativa, atrás apenas da sardinha e da corvina (SILVA et al., 2005). Essa espécie pode ser o único alvo da pesca de camarões (pesca monoespecífica) (FERNANDES et al., 2011), ou pode compartilhar os campos de pesca costeiros com outras espécies que compõe o desembarque pesqueiro e apresentam interesse comercial, tais como camarão barba-ruça (Artemesia longinaris Bate, 1888), camarão branco (Litopenaeus schmitti Burkenroad, 1936) e camarões rosa (Farfantepenaeus spp.).

Os crustáceos possuem exoesqueleto quitinoso e rígido, e seu crescimento se caracteriza por processo descontínuo e assintótico, ocorrendo periodicamente após o indivíduo realizar a ecdise. Os camarões peneídeos têm taxas de crescimento elevadas e ciclo de vida curto, com longevidade que varia de menos de 2,0 a 3,0 anos (LEITE JUNIOR et al., 2006; FERNANDES et al., 2011; DAVANSO et al., 2017). O dimorfismo sexual no tamanho corporal é comum nesses camarões. As fêmeas são em geral mais pesadas que os machos, e possuem maior tamanho de cefalotórax e abdômen relacionado as funções reprodutivas, correspondendo ao desenvolvimento do ovário e a produção de oócitos (HARTNOLL, 1982).

As relações morfométricas que envolvem peso e comprimento corporal são as mais utilizadas para determinar o crescimento relativo das espécies, permitindo não apenas estimar o peso médio em um determinado comprimento, mas também comparar a história de vida entre regiões e avaliar a dinâmica populacional (KING, 2007; FERREIRA et al., 2008; SEVERINO-RODRIGUES et al., 2016). Pressões antrópicas, tais como as pescarias, podem influenciar nas taxas de crescimento (KING, 2007; FONTELES FILHO, 2011). As variações nas relações morfométricas em camarões também refletem variações nas condições ambientais ao longo das áreas de distribuição, tais como temperatura da água e disponibilidade de alimento, e/ou pressões interespecíficas, como a competição por recursos, dessa forma, cada espécie pode contribuir para a regulação da população/estoque de outra espécie (PÉREZ-CASTAÑEDA et al., 2002; BISSARO et al., 2013).

O objetivo do estudo é ajustar e comparar as relações peso-comprimento de três espécies de camarões peneídeos (X. kroyeri, A. longinaris e L. schmitti) capturadas comercialmente em pescarias artesanais praticadas em seis portos de pesca no sudeste do Brasil. A relação peso-comprimento permite avaliar o crescimento relativo da população (ou estoque), e a principal questão do estudo é se a simpatria entre estoques de diferentes espécies de camarões influencia no seu crescimento relativo.

\section{METODOLOGIA}

Os camarões foram amostrados a partir de desembarques realizados em três portos de pesca do estado do Espírito Santo (Conceição da Barra, Vitória e Anchieta) e três portos do estado do Rio de Janeiro

\footnotetext{
${ }^{1}$ http://www.fao.org/fishery/species/2600/en

2 http://www.fiperj.rj.gov.br/index.php/main/relatorioanual
} 
(Atafona, Farol de São Tomé e Macaé), sudeste do Brasil (Figura 1). Os campos de pesca das embarcações que atuam a partir desses portos se localizam em áreas próximas a eles, até 20-30 m de profundidade, e a pesca é artesanal, praticada com redes de arrasto de fundo. Nos portos de Conceição da Barra, Vitória e Atafona, a pescaria comercial de camarão é monoespecífica, e somente $X$. kroyeri está presente nos desembarques. Por outro lado, em Anchieta, Farol de São Tomé e Macaé mais de uma espécie está presente e a pescaria de camarão é multiespecífica, com as espécies igualmente capturadas para fins comerciais (Tabela 1). Considerando que $X$. kroyeri está presente em todos os portos de pesca, essa espécie norteou as comparações do crescimento relativo na ausência e na presença de outros camarões. As amostragens foram realizadas entre junho e julho de 2017, a partir do volume total desembarcado. Durante a coleta foram selecionados 120 indivíduos de cada espécie, sendo 60 machos e 60 fêmeas (Tabela 1). Camarões machos e fêmeas foram distintos entre si pela presença de estruturas morfológicas externas que representam os caracteres sexuais secundários de camarões peneídeos: télico para fêmeas e petasma (órgão copulador) para machos (HARTNOLL, 1982).

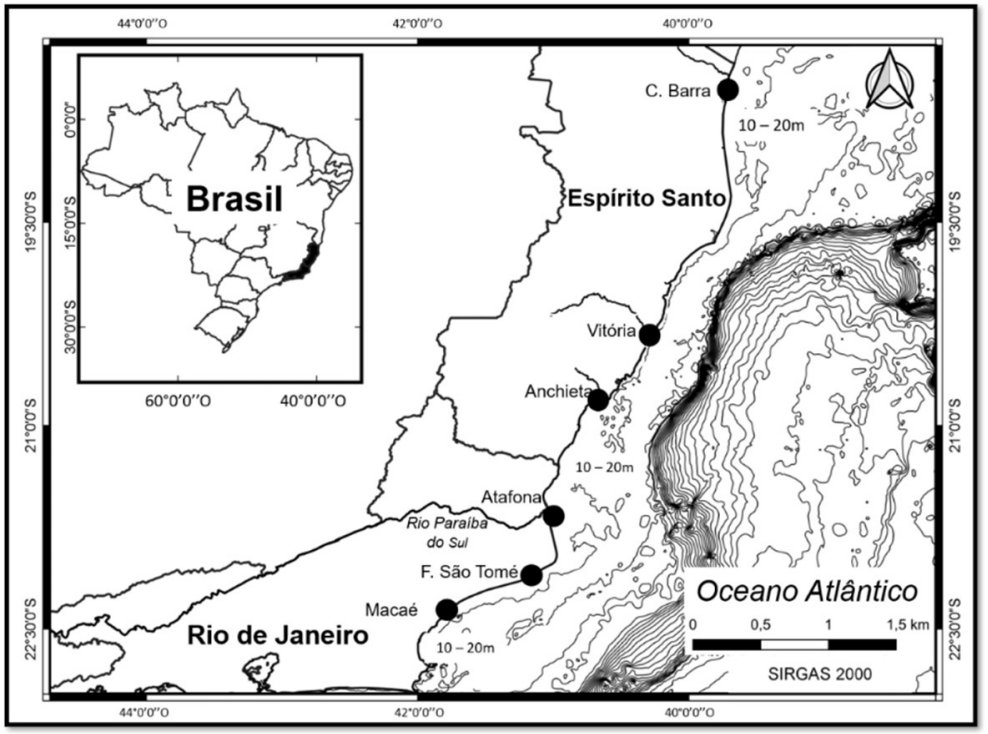

Figura 1: Localização dos seis portos de pesca no sudeste do Brasil, nos quais as espécies de camarões peneídeos foram amostradas.

Todos os indivíduos foram medidos em projeção retilínea quanto ao comprimento do cefalotórax com paquímetro manual $(0,1 \mathrm{~mm})$. A medida corresponde da margem do orbital posterior ao final da margem posterior do cefalotórax $(0,1 \mathrm{~mm})$. 0 peso total foi aferido em balança digital $(0,1 \mathrm{~g})$.

Todos os dados foram analisados no programa R (R CORE TEAM, 2020). As equações de regressão peso-comprimento $\left(\mathrm{P}=a^{*} C C^{b}\right)$ foram ajustadas para machos e fêmeas separadamente, e entre os gêneros combinados, com P correspondendo ao peso total (g) e CC ao comprimento da carapaça (mm). Os parâmetros da equação foram estimados pelo método dos mínimos quadrados: $a$ é o coeficiente linear e $b$ é o coeficiente angular ou alométrico.

O coeficiente alométrico $(b)$ define o tipo de crescimento relativo e indica o modo como uma população (ou estoque) investe no seu desenvolvimento, demonstrando se há maior investimento em aumento de peso ou comprimento. 0 valor de referência de $b$ é 3 , devido à relação cúbica entre essas 
medidas corporais. Se $b=3$, o crescimento é isométrico, e as partes do corpo comparadas aumentam na mesma proporção. Se $b<3$, o crescimento é alométrico negativo, ou seja, o peso aumenta a uma taxa relativamente menor que o comprimento. Quando $b>3$, o crescimento é alométrico positivo, e o peso aumenta a uma taxa relativamente maior quando comparado ao comprimento (FONTELES FILHO, 2011; SOUZA et al., 2019). O tipo de crescimento relativo dos camarões foi avaliado pelo teste t de Student em cada gênero (fêmea e macho) e espécie (gêneros combinados) para determinar a natureza isométrica da relação peso-comprimento $(b=3)$, com nível de significância de 0,05.

Análise de covariância (ANCOVA) avaliou as diferenças no valor do coeficiente alométrico considerando: i) estoques diferentes da mesma espécie (gêneros separados) e ii) gêneros dentro do mesmo estoque. A transformação logarítmica foi aplicada aos dados, atendendo aos pressupostos da ANCOVA. Peso total (log) foi considerado como a variável dependente, comprimento da carapaça (log) como covariável, e gênero e porto de pesca como fatores principais. Os coeficientes de determinação $r^{2}(>0.90)$ indicaram a qualidade do ajuste da ANCOVA.

\section{RESULTADOS}

Em geral, os camarões com as maiores dimensões de peso total e comprimento do cefalotótax pertenceram as espécies L. schmitti, X. kroyeri e A. longinaris, nessa ordem (Tabela 1). As médias do comprimento do cefalotórax entre os estoques analisados, considerando os gêneros combinados, variaram entre 21,0 e $21,3 \mathrm{~mm}$ para L. schmitti, 16,5 e 22,1 mm para X. kroyeri e 14,3 e 15,9 mm para A. longinaris. Em relação ao peso total, as médias variaram de 6,5 a 9,7 g (L. schmitti), 3,0 a 7,5 g (X. kroyeri) e 2,0 a 2,7 g (A. longinaris). Em todas as espécies e estoques, os indivíduos machos apresentaram dimensões menores em comparação as fêmeas (Tabela 1).

Tabela 1: Valores médios de comprimento do cefalotórax $(\mathrm{mm})$ e peso $(\mathrm{g})$ de camarões peneídeos capturados na costa sudeste do Brasil. F: fêmea, M: macho, F+M: gêneros combinados, EP: erro padrão, Min: Mínimo, Max: Máximo.

\begin{tabular}{|c|c|c|c|c|c|c|}
\hline \multirow[t]{2}{*}{ Espécie } & \multirow[t]{2}{*}{ Porto } & \multirow[t]{2}{*}{ Gênero } & \multicolumn{2}{|c|}{ Comprimento da carapaça (mm) } & \multicolumn{2}{|l|}{ Peso total (g) } \\
\hline & & & Média \pm EP & Min-Max & Média \pm EP & Min-Max \\
\hline \multirow[t]{18}{*}{ X. kroyeri } & C. Barra & $\mathrm{F}$ & $17,0 \pm 0,4$ & $10,5-23,2$ & $3,0 \pm 0,1$ & $0,6-7,1$ \\
\hline & 18오'S; 394' W & $\mathrm{M}$ & $16,0 \pm 0,3$ & $11,2-21,2$ & $3,0 \pm 0,1$ & $1,1-5,9$ \\
\hline & & $\mathrm{F}+\mathrm{M}$ & $16,5 \pm 0,2$ & $10,5-23,2$ & $3,0 \pm 0,1$ & $0,6-7,1$ \\
\hline & Vitória & $\mathrm{F}$ & $19,0 \pm 0,6$ & $12,0-30,0$ & $5,2 \pm 0,4$ & $1,3-14,6$ \\
\hline & 203's; 403’'W & $\mathrm{M}$ & $18,0 \pm 0,4$ & $13,0-23,0$ & $4,4 \pm 0,2$ & $1,6-8,2$ \\
\hline & & $\mathrm{F}+\mathrm{M}$ & $18,3 \pm 0,4$ & $12,0-30,0$ & $5,5 \pm 0,2$ & $1,3-14,6$ \\
\hline & Anchieta & $F$ & $17,0 \pm 0,6$ & $10,0-30,0$ & $4,3 \pm 0,3$ & $1,0-14,7$ \\
\hline & $20 \div 48^{\prime} \mathrm{S} ; 40 \div 38^{\prime} \mathrm{W}$ & $\mathrm{M}$ & $16,0 \pm 0,4$ & $10,0-23,0$ & $3,3 \pm 0,2$ & $1,0-8,8$ \\
\hline & & $\mathrm{F}+\mathrm{M}$ & $19,6 \pm 0,4$ & $10,0-30,0$ & $3,8 \pm 0,2$ & $1,0-14-7$ \\
\hline & Atafona & $\mathrm{F}$ & $22,0 \pm 0,5$ & $15,0-31,0$ & $6,9 \pm 0,3$ & $2,3-14,6$ \\
\hline & $21^{\circ} 37^{\prime} \mathrm{S} ; 41^{\circ} 00^{\prime} \mathrm{W}$ & $\mathrm{M}$ & $19,0 \pm 0,3$ & $14,0-25,0$ & $5,0 \pm 0,2$ & $2,1-9,2$ \\
\hline & & $\mathrm{F}+\mathrm{M}$ & $20,6 \pm 0,3$ & $14,0-31,0$ & $6,0 \pm 0,2$ & $2,1-14,6$ \\
\hline & F. São Tomé & $\mathrm{F}$ & $24,0 \pm 0,3$ & $20,0-30,0$ & $8,9 \pm 0,3$ & $5,3-14,1$ \\
\hline & $22^{\circ} 02^{\prime} \mathrm{S} ; 4^{\circ} 02^{\prime} \mathrm{W}$ & $\mathrm{M}$ & $20,0 \pm 0,5$ & $15,0-25,0$ & $5,9 \pm 0,3$ & $2,4-11,1$ \\
\hline & & $\mathrm{F}+\mathrm{M}$ & $22,1 \pm 0,3$ & $15,0-30,0$ & $7,5 \pm 0,2$ & $2,4-14,1$ \\
\hline & Macaé & $\mathrm{F}$ & $23,0 \pm 0,6$ & $16,4-33,2$ & $8,3 \pm 0,5$ & $3,1-18,0$ \\
\hline & $22^{\circ} 22^{\prime} \mathrm{S} ; 41^{\circ} 47^{\prime} \mathrm{W}$ & $\mathrm{M}$ & $21,0 \pm 0,3$ & $16,3-25,0$ & $6,7 \pm 0,2$ & $2,9-10,9$ \\
\hline & & $\mathrm{F}+\mathrm{M}$ & $22,1 \pm 0,4$ & $16,3-33,2$ & $7,5 \pm 0,3$ & $2,4-18,0$ \\
\hline \multirow[t]{2}{*}{ L. schmitti } & Anchieta & $\mathrm{F}$ & $21,0 \pm 0,5$ & $13,2-33,1$ & $9,4 \pm 0,6$ & $1,6-21,5$ \\
\hline & $20 \div 48^{\prime} \mathrm{S} ; 40038^{\prime} \mathrm{W}$ & $\mathrm{M}$ & $22,0 \pm 0,4$ & $14,9-29,9$ & $10,0 \pm 0,5$ & $2,7-21,5$ \\
\hline
\end{tabular}




\begin{tabular}{|l|l|l|l|l|l|l|}
\hline & & $F+M$ & $21,3 \pm 0,4$ & $13,2-33,1$ & $9,7 \pm 0,4$ & $1,6-22,5$ \\
\hline & Macaé & F & $23,0 \pm 0,7$ & $13,4-33,3$ & $8,1 \pm 0,6$ & $1,5-19,1$ \\
\hline \multirow{2}{*}{ A. longinaris } & $22^{\circ} 22^{\prime} \mathrm{S} ; 41^{\circ} 47^{\prime} \mathrm{W}$ & $\mathrm{M}$ & $19,0 \pm 0,2$ & $16,1-22,0$ & $4,8 \pm 0,1$ & $3,0-7,0$ \\
\hline & & $\mathrm{F}+\mathrm{M}$ & $21,0 \pm 0,4$ & $13,4-33,3$ & $6,5 \pm 0,3$ & $1,5-19,1$ \\
\hline & F. São Tomé & $\mathrm{F}$ & $15,0 \pm 0,2$ & $10,7-18,9$ & $2,2 \pm 0,1$ & $1,1-4,1$ \\
\hline & $22^{\circ} 02^{\prime} \mathrm{S} ; 41^{\circ} 02^{\prime} \mathrm{W}$ & $\mathrm{M}$ & $13,0 \pm 0,2$ & $10,6-17,4$ & $1,7 \pm 0,1$ & $1,1-3,3$ \\
\hline & & $\mathrm{F}+\mathrm{M}$ & $14,3 \pm 0,2$ & $10,6-18,9$ & $2,0 \pm 0,1$ & $1,1-4,1$ \\
\hline & Macaé & $\mathrm{F}$ & $17,0 \pm 0,4$ & $9,9-23,6$ & $3,2 \pm 0,2$ & $0,8-6,1$ \\
\hline & $22^{\circ} 22^{\prime} \mathrm{S} ; 41^{\circ} 47^{\prime} \mathrm{W}$ & $\mathrm{M}$ & $14,0 \pm 0,2$ & $10,0-17,7$ & $2,1 \pm 0,1$ & $0,7-3,5$ \\
\hline & & $\mathrm{F}+\mathrm{M}$ & $15,9 \pm 0,3$ & $9,9-23,6$ & $2,7 \pm 0,1$ & $0,7-6,1$ \\
\hline
\end{tabular}

Os valores do coeficiente alométrico determinados nas equações indicam uma alometria negativa ( $b$ <3) em relação ao crescimento relativo dos camarões para todas as espécies, gêneros e estoques estudados (Tabela 2). Os resultados da ANCOVA indicam que os coeficientes alométricos não diferiram entre os estoques da mesma espécie ao longo dos portos de pesca, e nem entre fêmeas e machos considerando o mesmo estoque $(p>0.05)$, com exceção de $X$. kroyeri no Farol de São Tomé (Tabela 2). Nesse porto de pesca, os machos mantêm o crescimento alométrico negativo, mas o valor de $b(2,96)$ foi significativamente maior em comparação às fêmeas $(2,24)(p<0,05)$ (Tabela 2, Figura 2).

Tabela 2: Relação comprimento-peso das espécies de camarões estudadas em portos de pesca do sudeste do Brasil. F: fêmea, $\mathrm{M}$ : macho, $\mathrm{F}+\mathrm{M}$ : gêneros combinados, P: peso total (g), CC: comprimento do cefalotórax $(\mathrm{mm})$. Valores de $p$ indicam se o coeficiente alométrico $(b)$ é diferente de 3.

\begin{tabular}{|c|c|c|c|c|c|c|c|}
\hline Espécie & Porto & Gênero & Equação & $r^{2}$ & $\boldsymbol{b}$ & Alometria & $p$ \\
\hline \multirow[t]{18}{*}{ X. kroyeri } & C. Barra & $\mathrm{F}$ & $\mathrm{P}=0,0027, \mathrm{CC}^{2,47}$ & 0,89 & 2,47 & negativa & $<0,0001$ \\
\hline & $18 \div 35^{\prime} \mathrm{S} ; 39 \circ 44^{\prime} \mathrm{W}$ & $\mathrm{M}$ & $P=0,001, C C^{2,83}$ & 0,94 & 2,83 & negativa & $<0,0001$ \\
\hline & & $\mathrm{F}+\mathrm{M}$ & $P=0,0018, C C^{2,62}$ & 0,91 & 2,62 & negativa & $<0,0001$ \\
\hline & Vitória & $\mathrm{F}$ & $P=0,0039, C C^{2,40}$ & 0,93 & 2,40 & negativa & $<0,0001$ \\
\hline & $20 \div 31^{\prime} \mathrm{S} ; 40 \div 30^{\prime} \mathrm{W}$ & $M$ & $P=0,0019, C C^{2,68}$ & 0,93 & 2,68 & negativa & $<0,0001$ \\
\hline & & $\mathrm{F}+\mathrm{M}$ & $P=0,0036, C C^{2,45}$ & 0,93 & 2,45 & negativa & $<0,0001$ \\
\hline & Anchieta & $\mathrm{F}$ & $P=0,0035, C C^{2,44}$ & 0,98 & 2,44 & negativa & $<0,0001$ \\
\hline & $20 \div 48^{\prime} S ; 40 \div 38^{\prime} W$ & $M$ & $P=0,0019, C C^{2,67}$ & 0,97 & 2,67 & negativa & $<0,0001$ \\
\hline & & $\mathrm{F}+\mathrm{M}$ & $\mathrm{P}=0,0031, \mathrm{CC}^{2,49}$ & 0,97 & 2,49 & negativa & $<0,0001$ \\
\hline & Atafona & $\mathrm{F}$ & $P=0,0034, C C^{2,44}$ & 0,97 & 2,44 & negativa & $<0,0001$ \\
\hline & $21^{\circ} 37^{\prime} \mathrm{S} ; 41^{\circ} 00^{\prime} \mathrm{W}$ & $M$ & $P=0,0021, C C^{2,63}$ & 0,95 & 2,63 & negativa & $<0,0001$ \\
\hline & & $\mathrm{F}+\mathrm{M}$ & $P=0,0037, C C^{2}, 42$ & 0,95 & 2,42 & negativa & $<0,0001$ \\
\hline & F. São Tomé & $\mathrm{F}$ & $P=0,0069, C C^{2}, 24$ & 0,92 & 2,24 & negativa & $<0,0001$ \\
\hline & $22^{\circ} 02^{\prime} \mathrm{S} ; 41^{\circ} 02^{\prime} \mathrm{W}$ & $\mathrm{M}$ & $P=0,0008, C C^{2}, 96$ & 0,92 & 2,96 & negativa & 0,0001 \\
\hline & & $\mathrm{F}+\mathrm{M}$ & $P=0,0024, C C^{2,59}$ & 0,93 & 2,59 & negativa & $<0,0001$ \\
\hline & Macaé & $\mathrm{F}$ & $P=0,0061, C C^{2,27}$ & 0,95 & 2,27 & negativa & $<0,0001$ \\
\hline & $22^{\circ} 22^{\prime} \mathrm{S} ; 41^{\circ} 47^{\prime} \mathrm{W}$ & M & $P=0,002, C C^{2,65}$ & 0,84 & 2,65 & negativa & $<0,0001$ \\
\hline & & $\mathrm{F}+\mathrm{M}$ & $P=0,0054, C C^{2,32}$ & 0,91 & 2,32 & negativa & $<0,0001$ \\
\hline \multirow[t]{6}{*}{ L. schmitti } & Anchieta & $\mathrm{F}$ & $P=0,0018, C C^{2,78}$ & 0,92 & 2,78 & negativa & $<0,0001$ \\
\hline & $20 \div 48^{\prime} \mathrm{S} ; 40 \div 38^{\prime} \mathrm{W}$ & $M$ & $P=0,0041, C C^{2,51}$ & 0,89 & 2,51 & negativa & $<0,0001$ \\
\hline & & $\mathrm{F}+\mathrm{M}$ & $P=0,0025, C C^{2}, 68$ & 0,91 & 2,68 & negativa & $<0,0001$ \\
\hline & Macaé & $\mathrm{F}$ & $P=0,0019, C C^{2,63}$ & 0,94 & 2,63 & negativa & $<0,0001$ \\
\hline & $22^{\circ} 22^{\prime} \mathrm{S} ; 41^{\circ} 47^{\prime} \mathrm{W}$ & M & $P=0,009, C C^{2,13}$ & 0,71 & 2,13 & negativa & $<0,0001$ \\
\hline & & $\mathrm{F}+\mathrm{M}$ & $P=0,003, C C^{2,49}$ & 0,92 & 2,49 & negativa & $<0,0001$ \\
\hline \multirow[t]{6}{*}{ A. longinaris } & F. São Tomé & $\mathrm{F}$ & $P=0,003, C C^{2,42}$ & 0,84 & 2,42 & negativa & $<0,0001$ \\
\hline & $22^{\circ} 02^{\prime} \mathrm{S} ; 4^{\circ} 02^{\prime} \mathrm{W}$ & $M$ & $P=0,0058, C C^{2}, 18$ & 0,75 & 2,18 & negativa & $<0,0001$ \\
\hline & & $\mathrm{F}+\mathrm{M}$ & $P=0,0045, C C^{2,27}$ & 0,83 & 2,27 & negativa & $<0,0001$ \\
\hline & Macaé & $\mathrm{F}$ & $P=0,0032, C^{2}, 39$ & 0,95 & 2,39 & negativa & $<0,0001$ \\
\hline & $22^{\circ} 22^{\prime} \mathrm{S} ; 41^{\circ} 47^{\prime} \mathrm{W}$ & $M$ & $P=0,0022, C C^{2,54}$ & 0,90 & 2,54 & negativa & $<0,0001$ \\
\hline & & $\mathrm{F}+\mathrm{M}$ & $P=0,0031, C C^{2}, 42$ & 0,94 & 2,42 & negativa & $<0,0001$ \\
\hline
\end{tabular}



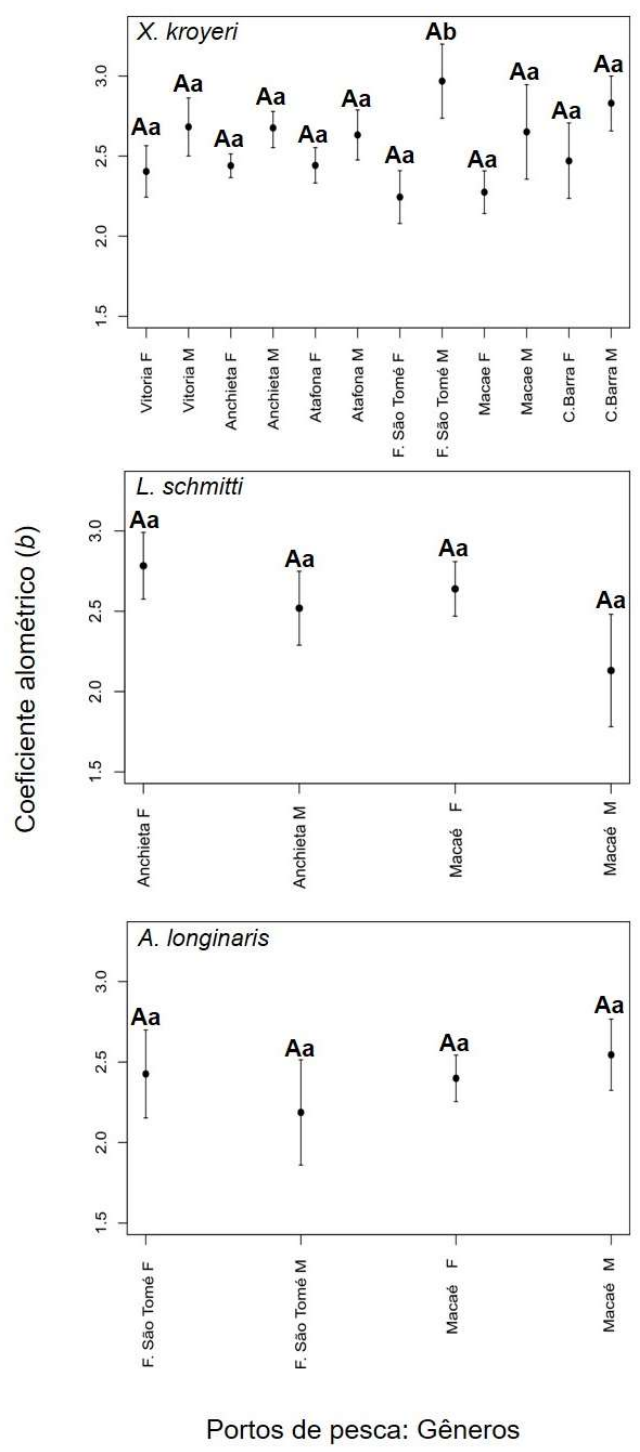

Figura 2: Comparação dos coeficientes alométricos $(b)$ da relação comprimento-peso nas espécies de camarões estudadas em portos de pesca do sudeste do Brasil. F: Fêmea, M: Machos. Barras representam intervalos de confiança de $95 \%$. Letras maiúsculas representam igualdade/diferença do mesmo gênero entre os estoques (portos de pesca), e letras minúsculas representam igualdade/diferença entre os gêneros dentro do mesmo estoque.

\section{DISCUSSÃO}

A simpatria entre os camarões peneídeos não influenciou no seu crescimento relativo nos portos de pesca estudados. Os coeficientes alométricos são similares entre os estoques da mesma espécie, tanto para fêmeas quanto para machos, independentemente da presença de outra espécie no campo de pesca. Para $X$. kroyeri, que se distribui nos seis portos de pesca estudados, o crescimento relativo de fêmeas, machos e gêneros combinados foi semelhante tanto onde a pesca é monoespecífica (Conceição da Barra, Vitória e Atafona) quanto na presença de outras espécies de camarões (Anchieta, Farol de São Tomé e Macaé).

$\mathrm{Na}$ relação peso-comprimento, o coeficiente alométrico geralmente não varia ao longo do ano, e pode ser considerado como valor médio do crescimento relativo anual das espécies (SANTOS et al., 2002; ANDREU-SOLER et al., 2006). Dessa forma, o fato dos camarões serem coletados em amostragem única (junho-julho 2017) não interferiu nos resultados. Além disso, os coeficientes de determinação $\left(r^{2}\right)$ dos ajustes entre as medidas de peso total e comprimento do cefalotórax foram, em geral, acima de 0.90 , corroborando 
a consistência dos resultados (Tabela 2).

Todas as espécies de camarões demonstraram forte alometria negativa quanto ao crescimento, com o peso corporal aumentando a uma taxa menor em comparação ao comprimento do cefalotórax. O mesmo padrão é registrado quando indivíduos fêmeas e machos são analisados separadamente. Em espécies de crustáceos, a alometria negativa na relação peso-comprimento pode estar relacionada a baixa disponibilidade de presas, o que é característico em águas oligotróficas (THESSALOU-LEGAKI et al., 2005; SOUSA et al., 2019). Carvalho et al. (2019) analisaram as relações morfométricas em indivíduos de Farfantepenaeus brasiliensis e F. paulensis provenientes de duas áreas próximas no estado do Rio de Janeiro, e registraram variações espaciais significativas nos coeficientes alométricos, relacionando-as com variações na quantidade de alimento disponível. As características do hábitat relacionadas a disponibilidade de alimento poderiam justificar, ao menos em parte, a alometria negativa registrada nos estoques analisados.

Esse estudo apresenta o primeiro ajuste da relação peso-comprimento em estoques naturais de $L$. schmitti da costa dos estados do Espírito Santo (Anchieta) e Rio de Janeiro (Macaé). O gênero Litopenaeus apresenta alto valor comercial, e L. schmitti é a única espécie do gênero com distribuição natural associada a costa brasileira, considerada promissora para a carcinocultura no país (FUGIMURA et al., 2015). No entanto, suas populações naturais são impactadas pela pressão de pescarias costeiras e pela introdução do camarão branco do Pacífico, L. vannamei, a partir de cultivos comerciais intensivos, principalmente no nordeste do Brasil (MAGGIONI et al., 2003; REGO et al., 2018).

A costa brasileira pode ser subdividida em região tropical $\left(5^{\circ} \mathrm{N}\right.$ até $\left.23^{\circ} \mathrm{S}\right)$ e subtropical $\left(23^{\circ} \mathrm{S}\right.$ até $\left.35^{\circ} \mathrm{S}\right)$, e dentro desse intervalo é reconhecida uma zona de transição marinha entre 18ㅇ-23ㄴ, na qual as

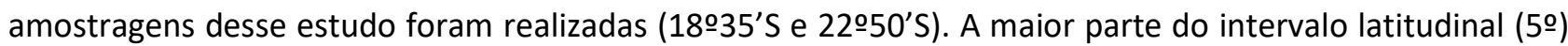
relacionado a zona de transição marinha é banhado pelas águas oligotróficas e quentes da Corrente do Brasil, mas no limite sul desse intervalo há ressurgência sazonal da Água Central do Atlântico Sul - ACAS (VALENTIN et al., 1993). A ressurgência da ACAS, que é uma massa d'água fria $\left(<20^{\circ} C\right)$ e rica em nutrientes, se dá predominantemente nos meses de verão, aumentando a produtividade primária costeira na região de Cabo Frio ( 23S), com influência em regiões próximas (COELHO-SOUZA et al., 2012). Isso inclui os campos de pesca das embarcações que atuam nos portos de Macaé e Farol de São Tomé, e justifica a presença de $A$. longinaris em quantidades suficientes para fazer parte de desembarques comerciais, uma vez que a espécie é característica de águas mais frias (BOOS et al., 2016; SANCINETTI et al., 2015; DAVANSO et al., 2017).

Apesar das relações peso-comprimento apresentarem comportamento semelhante entre as espécies (X. kroyeri, A. longinaris e L. schimitti), gêneros (fêmeas e machos) e estoques analisados (Conceição da Barra, Vitória, Anchieta, Atafona, Farol de São Tomé e Macaé), notam-se diferenças no tamanho corporal dos camarões (Tabela 1). Diferenças específicas são esperadas, pois as dimensões corporais e as taxas de crescimento podem diferir bastante dentro do mesmo clado (família Penaeidae) (HARTNOLL, 1982). O desenvolvimento morfológico diferenciado entre fêmeas e machos de camarões peneídeos é resultado do investimento diferenciado na reprodução, com fêmeas geralmente maiores e mais pesadas (KING, 2007), conforme observado em todas as espécies/estoques analisados. Além disso, a maior longevidade das fêmeas 
em comparação aos machos estende o período de crescimento, o que também justifica as maiores dimensões corporais, pois o crescimento da maioria dos crustáceos é continuado ao longo da vida (VOGT, 2012; DAVANSO et al., 2017).

As variações no tamanho corporal entre os diferentes estoques de camarão serão discutidas apenas para X. kroyeri, cujas amostragens incluíram todos os portos de pesca e, portanto, uma distribuição maior em relação as outras espécies. De acordo com o paradigma latitudinal estabelecido pela regra de Bergmann para animais homeotérmicos (BERGMANN, 1847), e depois estendida para animais heterotérmicos, incluindo crustáceos (TIMOFEEV, 2001), o tamanho corporal em animais é maior em regiões mais frias (maiores latitudes) em comparação a regiões mais quentes (menores latitudes). Portanto, espera-se que quando a mesma espécie se distribui entre diferentes gradientes latitudinais haja variação no seu tamanho corporal devido as variações de temperatura/latitude. Os dados de comprimento do cefalotórax e peso total de $X$. kroyeri apresentados na tabela 1 indicam que, em princípio, a espécie se enquadraria na regra de Bergmann.

Variações ambientais que ocorrem temporalmente, tais como flutuação na temperatura da água e na produtividade primária, podem afetar a base das cadeias tróficas marinhas e a disponibilidade de recursos locais (LEWANDOWSKA et al., 2012), com reflexos imediatos no crescimento dos camarões que são consumidores secundários (DI BENEDITTO et al., 2012). O tamanho corporal de $X$. kroyeri apresentou variações em intervalos temporais distintos, ao longo de um intervalo espacial (latitudinal) sobreposto. Em 2009, Bissaro et al. (2013) coletaram indivíduos em quatro portos de pesca localizados entre 17ำ4’S e 22ํำ'S, registrando redução no tamanho corporal com o aumento da latitude. Em 2017, oito anos depois, o presente estudo coletou indivíduos em seis portos de pesca entre $18^{\circ} 35^{\prime}$ S e $22^{\circ}=50^{\prime} \mathrm{S}$, e verificou aumento no tamanho corporal com o aumento da latitude (regra de Bergmann). As condições ambientais nos locais de coleta, tais como temperatura da água, salinidade e concentração de clorofila a (indicativo da produtividade primária) não foram medidas em 2009 e nem em 2017 para possíveis relações com a disponibilidade de recursos alimentares; no entanto, inferências explicativas para essas diferenças baseadas no ciclo de vida, na fisiologia e no comportamento da espécie podem ser feitas. Xiphopenaeus kroyeri tem longevidade média de 2,0 anos, pelo menos dois picos reprodutivos principais anuais e taxa de crescimento elevada (FERNANDES et al., 2011; DAVANSO et al., 2017). A espécie tolera águas com temperaturas que variam entre $15^{\circ} \mathrm{C}$ e $30^{\circ} \mathrm{C}$ e salinidade entre 9 e 36, e apresenta elevada plasticidade alimentar (BOOS et al., 2016; WILLEMS et al., 2016). A partir dessas características, presume-se que $X$. kroyeri é capaz de se adaptar rapidamente as variações em seu hábitat, inclusive com alterações em seu crescimento relativo.

\section{CONCLUSÕES}

Apesar da simpatria não ser uma condição que tenha influência sobre o crescimento relativo de $X$. kroyeri, A. longinaris e L. schimitti ao longo dos portos de pesca estudados, dados prévios sobre $X$. kroyeri, por exemplo, demonstram que mudanças no tamanho e no desenvolvimento corporal podem ocorrer ao longo do tempo na mesma escala espacial. Dessa forma, recomenda-se o acompanhamento regular dos estoques para avaliar o desenvolvimento dos camarões no longo prazo como forma de monitorar a sua 
dinâmica de crescimento, principalmente pelo fato desses organismos serem importantes para o equilíbrio das cadeias tróficas locais e para a economia das comunidades litorâneas do sudeste do Brasil.

AGRADECIMENTOS: Ao Conselho Nacional de Desenvolvimento Científico e Tecnológico - CNPq (305.217/2017-8), à Fundação Carlos Chagas Filho de Amparo à Pesquisa do Estado do Rio de Janeiro - FAPERJ (E-26/202.770/2017; E-26/210.064/2018), e à Coordenação de Aperfeiçoamento de Pessoal de Nível Superior - CAPES (Código de financiamento 001).

\section{REFERÊNCIAS}

ANDREU-SOLER, A.; OLIVA-PATERNA, F. J.; TORRALVA, M.. A review of length-weight relationships of fish from the Segura River basin (SE Iberian Peninsula). Journal of Applied Ichthyology, v.22, n.4, p.295-296, 2006. DOI: https://doi.org/10.1111/j.1439-0426.2006.00719.x

BERGMANN, C.. Über die verhältnisse der wärmeökonomie der thiere zu ihrer grösse. Göttinger Studien, v.3, p.595-708, 1847.

BISSARO, F. G.; GOMES, JUNIOR, J. L.; DI BENEDITTO, A. P. $M$.. Morphometric variation in the shape of the cephalothorax of shrimp Xiphopenaeus kroyeri on the east coast of Brazil. Journal of the Marine Biological Association of United Kingdom, v.93, p.683-691, 2013. DOI: https://doi.org/10.1017/S0025315412000409

BOOS, H.; COSTA, R. C.; SANTOS, R. A. F.; DIAS NETO, J.; SEVERINO-RODRIGUES, E.; RODRIGUES, L. F.; D'INCAO, F.; IVO, C. T. C.; COELHO, P. A.. Avaliação dos camarões peneídeos (Decapoda: Penaeidae). In: PINHEIRO, M.; BOOS, H.. Livro vermelho dos crustáceos do Brasil: Avaliação 20102014. Porto Alegre: Sociedade Brasileira de Carcinologia, 2016. p.300-317.

CARVALHO, C. D.; KEUNECKE, K. A.; LAVRADO, H.

P.. Morphometric variation in pink shrimp populations at Rio de Janeiro coast (SE Brazil): are they really similar in closer areas?. Anais da Academia Brasileira de Ciências, v.91, n.2, p.e20180252, 2019. DOI: https://doi.org/10.1590/0001$\underline{3765201920180252}$

COELHO-SOUZA, S. A.; LÓPEZ, M. S.; GUIMARÃES, J. R. D.; COUTINHO, R.; CANDELLA, R. N.. Biophysical interactions in the Cabo Frio upwelling system, southeastern Brazil. Brazilian Journal of Oceanography, v.60, p.353-365, 2012. DOI: $\underline{\text { https://doi.org/10.1590/S1679-87592012000300008 }}$

DAVANSO, T. M.; HIROSE, G. L.; HERRERA, D. R.; FRANSOZO, A.; COSTA, R. C.. Does the upwelling phenomenon influence the population dynamics and management of the seabob shrimp Xiphopenaeus kroyeri (Heller, 1862) (Crustacea, Penaeidae)? Hydrobiologia, v.795, n.1, 295-311. 2017. DOI: https://doi.org/10.1007/s10750-017-3152-0

DI BENEDITTO, A. P. M.; BITTAR, V. T.; CAMARGO, P. B.; REZENDE, C. E.; KEHRIG, H. A.. Mercury and nitrogen isotope in a marine species from a tropical coastal food web. Archives of Environmental Contamination and Toxicology, v.62, p.264-271, 2012. DOI: https://doi.org/10.1007/s00244$\underline{011-9701-z}$
FERNANDES, L. P.; SILVA, A. C.; JARDIM, L. P.; KEUNECKE, K. A.; DI BENEDITTO, A. P. M.. Growth and recruitment of the atlantic seabob shrimp, Xiphopenaeus kroyeri (Heller, 1862) (Decapoda, Penaeidae), on the coast of Rio de Janeiro, southeastern Brazil. Crustaceana, v.84, p.1465-1480, 2011. DOI: https://doi.org/10.1163/156854011X605765

FERREIRA, S.; SOUSA, R.; DELGADO, J.; CARVALHO, D.; CHADA, T.. Weight-length relationships for demersal fish species caught off the Madeira archipelago (eastern-central Atlantic). Journal of Applied Ichthyology, v.24, n.1, p.93-95, 2008. DOI: https://doi.org/10.1111/j.14390426.2007.01027.x

FONTELES FILHO, A. A.. Oceanografia, biologia e dinâmica populacional de recursos pesqueiros. Fortaleza: Expressão, 2011.

FUGIMURA, M. M. S.; FLOR, H. R.; WASIELESKY JUNIOR, W.; OSHIRO, L. M. Y.. Criação do camarão Litopenaeus schmitti com diferentes salinidades e níveis de proteína na dieta em sistemas de bioflocos. Boletim do Instituto de Pesca, v.41, n.4, p.865-876, 2015.

GILLET, R.. Global study of shrimp fisheries. FAO Fisheries Technical Paper. № 475. Rome: FAO, 2008.

HARTNOLL, R. G.. Growth. In: BLISS, D.. The biology of Crustacea. New York: Academic Press, 1982. p.111-185.

KING, M. G.. Fisheries biology, assessment and management. Oxford: Blackwell Science, 2007.

LEITE JUNIOR, N. O.; PETRERE JUNIOR, M.. Crescimento e mortalidade dos camarões-rosa Farfantepenaeus brasiliensis Latreille, 1970 e F. paulensis Pérez-Farfante, 1967 no sudeste do Brasil. Revista Brasileira de Biologia, v.66, n.2, p.523-536, 2006. DOI: https://doi.org/10.1590/S1519$\underline{69842006000300019}$

LEWANDOWSKA, A. M.; BREITHAUPT, P.; HILLEBRAND, H.; HOPPE, H.-G.; JÜRGENS, K.; SOMMER, U.. Responses of primary productivity to increased temperature and phytoplankton diversity. Journal of Sea Research, v.72, p.8793, 2012.

MAGGIONI, R.; ROGERS, A. D.; MACLEAN, N.. Population structure of Litopenaeus schmitti (Decapoda: Penaeidae) from the Brazilian coast identified using six polymorphic microsatellite loci. Molecular Ecology, v.12, n.12, p.3213- 
3217, 2003. DOI: https://doi.org/10.1046/j.1365294X.2003.01987.X

PÉREZ-CASTAÑEDA, R.; DEFEO, O.. Morphometric relationships of penaeid shrimps in a coastal lagoon: Spatiotemporal variability and management implications.

Estuaries, v.25, n.2, p.282-287, 2002. DOI: https://doi.org/10.1007/BF02691315

R CORE TEAM. R: A language and environment for statistical computing. Viena: R Foundation for Statistical Computing, 2020.

REGO, M. A. S.; SABBAG, O. J.; SOARES, R. B.; PEIXOTO, S.. Technical efficiency analysis of marine shrimp farming (Litopenaeus vannamei) in biofloc and conventional systems: A case study in northeastern Brazil. Anais da Academia Brasileira de Ciências, v.90, n.4, p.3705-3716, 2018. DOI: https://doi.org/10.1590/0001-3765201820170953

SANTOS, M. N.; GASPAR, M. B.; VASCONCELOS, P.; MONTEIRO, C. C.. Weight-length relationships for 50 selected fish species of the of the Algarve coast (southern Portugal). Fisheries Research, v.59, n.1-2, p.289-295, 2002.

SANCINETTI, G.; AZEVEDO, A.; CASTILHO, A.; FRANSOZO, A.; COSTA, R.. Population biology of the commercially exploited shrimp Artemesia longinaris (Decapoda: Penaeidae) in an upwelling region in the Western Atlantic: comparisons at different latitudes. Brazilian Journal of Biology, v.75, n.2, p.305-313, 2015. DOI: https://doi.org/10.1590/1519$\underline{6984.11813}$

SEVERINO-RODRIGUES, E.; FURQUIM, L. G.; GRAÇA-LOPES, R.; ALVES, P. M. F.. Crescimento relativo e tamanho na maturidade sexual do lagostim Metanephrops rubellus (Moreira, 1903) desembarcado no litoral do estado de São Paulo, Brasil. Boletim do Instituto de Pesca, v.42, n.2, p.431442, 2016.
SILVA, A. O. A.; CARNEIRO, M. H.; MENDONÇA, J. T.; SERVO, G. J. M.; BASTOS, G. C. C.; SILVA, S. O.; BATISTA, P. A.. Produção pesqueira marinha do estado de São Paulo no ano 2004. Série de Relatórios Técnicos do Instituto de Pesca, v.20, p.1-40, 2005

SOUSA, R.; GOUVEIA, L.; PINTO, A. R.; TIMÓTEO, V.; DELGADO, J.; HENRIQUES, P.. Weight-lenght relationship of six shrimp species caught off the Madeira Archipelago, northeastern Atlantic. Brazilian Journal of Biology, v.79, p.133-138, 2019. DOI: https://doi.org/10.1590/1519$\underline{6984.180731}$

THESSALOU-LEGAKI, M.; PETRAKIS, G.; CHILARI, A. Population structure and reproduction of the deep-water shrimp Plesionika martia (Decapoda: Pandalidae) from the eastern Ionian Sea. Journal of Crustacean Biology, v.25, n.2, p.233-241, 2005. DOI: https://doi.org/10.1651/C-2513

TIMOFEEV, S. F.. Bergmann's principle and deep-water gigantism in marine crustaceans. Biological Bulletin-US, v.28, p.646-650, 2001. DOI:

https://doi.org/10.1023/A:1012336823275

VALENTIN, J. L.; MONTEIRO-RIBAS, W. M.. Zooplankton community structure on the east-southeast Brazilian

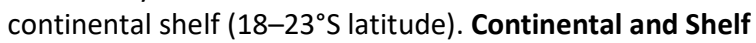
Research, v.13, n.4, p.407-424, 1993.

VOGT, G.. Ageing and longevity in the Decapoda (Crustacea): a review. Zoologischer Anzeiger, v.251, p.1-25, 2012. DOI: https://doi.org/10.1016/j.jcz.2011.05.003

WILLEMS, T.; BACKER, A.; KERKHOVE, T.; DAKRIET, N. N.; TROCH M.; VINCX, M.; HOSTENS, K.. Trophic ecology of Atlantic sea-bob shrimp Xiphopenaeus kroyeri: intertidal benthic microalgae support the subtidal food web off Suriname. Estuarine Coastal and Shelf Science, v.182, p.146157, 2016. DOI: https://doi.org/10.1016/j.ecss.2016.09.015

A CBPC - Companhia Brasileira de Produção Científica (CNPJ: 11.221.422/0001-03) detém os direitos materiais desta publicação. Os direitos referem-se à publicação do trabalho em qualquer parte do mundo, incluindo os direitos às renovaç̃oses, expansões e disseminações da contribuiç̃o, bem como outros direitos subsidiários. Todos os trabalhos publicados eletronicamente poderão posteriormente ser publicados em coletâneas impressas sob coordenação da Sustenere Publishing, da Companhia Brasileira de Produção Científica e seus parceiros autorizados. Os (as) autores (as) preservam os direitos autorais, mas não têm permissão para a publicação da contribuição em outro meio, impresso ou digital, em português ou em tradução. 